\title{
The Pattern of Protein Synthesis in spoIVC Mutants of Bacillus subtilis Resuspended in Sporulation Medium
}

\author{
By HASSIA BOSCHWITZ AND MICHAEL YUDKIN*† \\ Laboratory of Developmental and Molecular Biology, Department of Biological Chemistry, \\ Hebrew University of Jerusalem, Jerusalem, Israel
}

(Received 29 April 1983; revised 19 May 1983)

\begin{abstract}
Two spoIVC mutants of Bacillus subtilis were labelled with $\left[{ }^{35} \mathrm{~S}\right]$ methionine either at the time of resuspension in sporulation medium or 1,2 or $3 \mathrm{~h}$ later, and radioactive proteins were detected after cell extracts had been subjected to two-dimensional gel electrophoresis. The mutants completed almost all of the changes in protein synthesis that occur in the wild-type in these conditions. A heavily labelled protein was found in the mutants that has also been observed in a spo0 mutant but does not occur in the wild-type.
\end{abstract}

\section{INTRODUCTION}

Sporulation in Bacillus subtilis consists of a sequence of chemical, morphological and enzymic changes by which the vegetative cell is converted into a heat-resistant spore. These changes are completed in about $8 \mathrm{~h}$ at $37^{\circ} \mathrm{C}$. Many mutations are known that block sporulation. Characteristically the block occurs at a specific stage in the sequence, in such a way that the development of the spore appears to proceed normally as far as that stage. Such mutants are therefore known as spoII mutants, spoIV mutants, etc., depending on the stage of sporulation at which the block occurs (for review see Piggot \& Coote, 1976).

We have used the technique of two-dimensional gel electrophoresis to study changes in the pattern of protein synthesis in sporulation, and have identified some 75 proteins whose synthesis either starts or stops during the first $3 \mathrm{~h}$ of sporulation in the wild-type (Yudkin et al., 1982a). We have further shown that a spo0 mutation (which blocks sporulation extremely early) prevents about $50 \%$ of these changes, including many that are characteristic of the first hour of sporulation; by contrast a spoII mutation prevents only about $25 \%$ of the changes, very few of them in the first hour (Yudkin et al., 1982b). Since the original classification of the spoO and spoII mutants was based on relatively few enzymic tests, it is significant that they behaved by and large as expected when examined by the much more exhaustive and sensitive method of twodimensional gel electrophoresis.

These experiments did, however, reveal a few differences between the wild-type and the mutants that were not wholly expected. In particular, the mutants synthesized some proteins that were not seen at all in wild-type cultures (Yudkin et al., 1982 b).

We now report the results of similar studies with two spoIV mutants. These mutants appear, by the usual enzymic and morphological criteria, to be blocked at stage IV of sporulation, which is reached in wild-type cultures about $4 \mathrm{~h}$ after the beginning of sporulation. We therefore wanted to see whether they would behave like the wild-type when studied during the first $3 \mathrm{~h}$ of sporulation. U.K.

† Present address: Microbiology Unit, Department of Biochemistry, University of Oxford, Oxford OX13QU, 


\section{METHODS}

Organisms. Bacillus subtilis 168 MY2000.23 (pyrD1 lys-1 rfm spo-23) and MY2000.133 (pyrD1 lys-1 rfm spo133) are isogenic with the strains that we have studied previously (Yudkin et al., 1982a,b). They were constructed by transferring the two spoIVC mutations spo-23 and spo-133 from their original backgrounds into MY2002 (hisH pyrDI lys-1 rfm) by congression with his ${ }^{+}$.

Methods. All experimental techniques have been described by Yudkin et al. (1982a).

\section{RESULTS AND DISCUSSION}

We harvested cells of MY2000.23 and MY2000.133 from exponentially growing cultures, resuspended them in sporulation medium, and labelled with $\mathrm{L}-\left[{ }^{35} S\right]$ methionine for 5 min either immediately after resuspension $\left(t_{0}\right)$ or at hourly intervals thereafter $\left(t_{1}, t_{2}\right.$ or $\left.t_{3}\right)$. We prepared cell extracts rapidly at low temperature and displayed the radioactive proteins by two-dimensional gel electrophoresis followed by fluorography. Although the mutations in these two strains, spo23 and spo-133, are in different cistrons in spoIVC (Dancer \& Mandelstam, 1981), we found that in practice we could detect no difference between the results with the two strains, and we shall refer to 'the mutants' without distinguishing them.

The pattern of proteins synthesized at $t_{0}$ in the mutants (Fig. 1) was indistinguishable from that of the wild-type that we have described earlier (Yudkin et al., 1982a).

We have previously shown that wild-type cultures labelled at $t_{1}$ differ in many respects from similar cultures labelled at $t_{0}$. Thirty-five proteins are synthesized at $t_{1}$ that were not synthesized at $t_{0}$, and conversely nine proteins that were being made at $t_{0}$ apparently cease to be made by $t_{1}$.

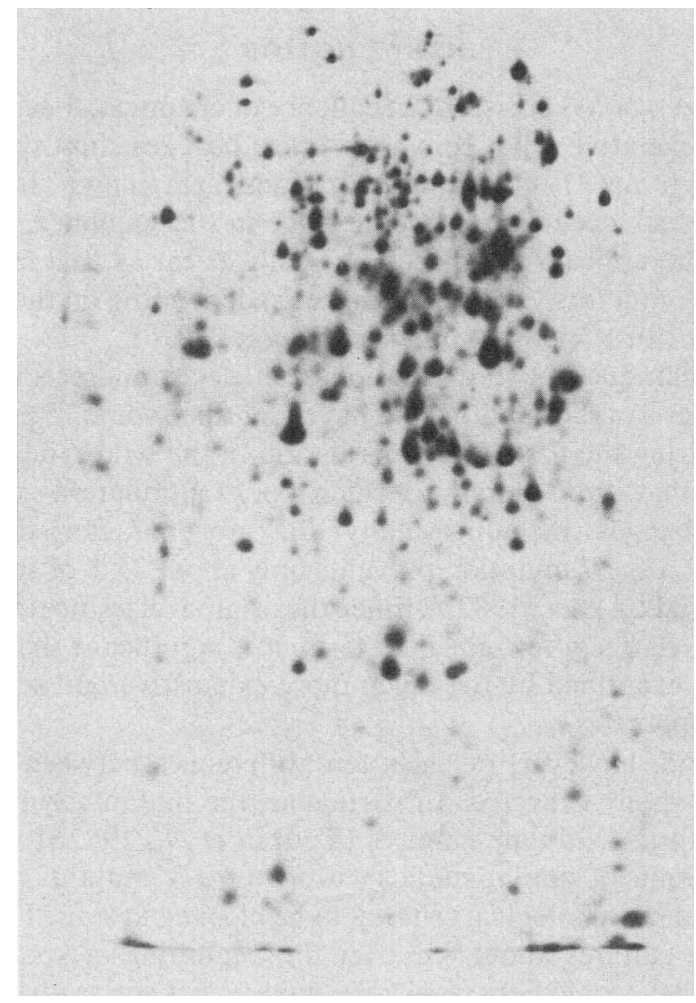

Fig. 1. Fluorogram of a two-dimensional separation of MY2000.23 labelled at $t_{0}$. In all the figures, the horizontal dimension corresponds to the separation by isoelectric focusing, with the more basic end being towards the left. The vertical dimension corresponds to the separation by SDS-PAGE, with the proteins of low apparent molecular weight being towards the bottom. 

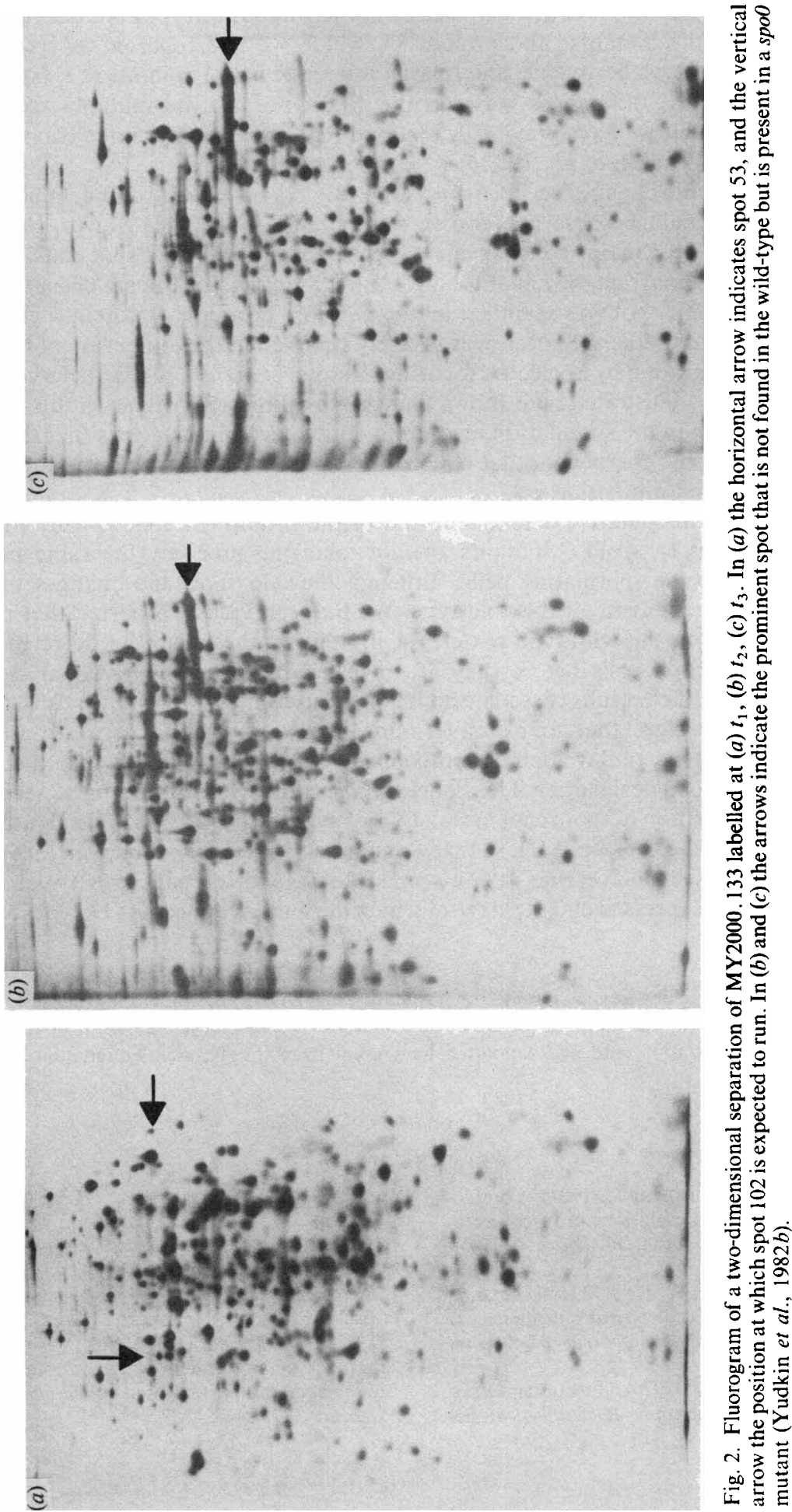
All of these 44 changes were found to occur also in the mutants, with two exceptions: protein 102, which we detected as a spot newly synthesized in the wild-type at $t_{1}$ (Yudkin et al., 1982a), was not observed in the mutants; and protein 53, which we had reported as ceasing to be synthesized in the wild-type between $t_{0}$ and $t_{1}$, was still made in the mutants at $t_{1}$ (see Fig. $2 a$ ). We doubt whether these differences between the wild-type and the mutants are of much significance: in particular we have previously reported that protein 102 is not always seen even in wild-type cultures (Yudkin et al., 1982b).

A further 30 detectable changes occur in wild-type cultures between $t_{1}$ and $t_{3}$ (Yudkin et al., 1982a). All of these could be observed also in the mutants (Fig. 2). However, the mutants differed from the wild-type in one conspicuous respect: a heavily labelled spot could be readily seen in patterns of proteins from the mutants labelled at $t_{2}$ and $t_{3}$ which did not occur in the wildtype (see Fig $2 b, c$ ). This spot was identical in position to a prominent protein seen in a spo0 mutant labelled at $t_{1}$, and, like the latter, was severely streaked in the dimension of the gel that corresponds to the separation by isoelectric focusing (Yudkin et al., 1982b). In fact a comparison of the four fluorograms illustrated here shows that the streaking of proteins in this dimension increases as incubation in the sporulation medium is continued. This suggests that the mutants may be synthesizing some component that renders their proteins less soluble and therefore less able to focus in the first-dimension gel.

The occurrence of this putative component and of the prominent spot already mentioned indicates that, even at $t_{2}$, spoIVC mutants are not following precisely the same pattern of development as wild-type sporulating cells, although they do show the changes in protein synthesis that are characteristic of the wild-type. We have previously reported that in a spoII mutant differences from the wild-type pattern of protein synthesis can be detected by twodimensional gel electrophoresis before they are revealed by more conventional techniques (Yudkin et al., 1982b). Such results cast some doubt on the usefulness of the normal classification of mutants, since they suggest that, for example, a 'spoIV' mutant, which is generally assumed to follow the normal pathway of development until stage IV is reached, may in fact diverge from the normal pattern a good deal earlier. This conclusion accords with the finding of Jenkinson $e t$ $a l$. (1981) that the formation of spore coat in stage $\mathrm{V}$ involves the deposition of proteins, many of which were made in stage II or stage III, so that a mutant classified as spoV may in fact possess a lesion in a gene expressed much earlier. It now seems likely that discrepancies between the stage at which a mutation is expressed and the stage at which the mutant appears to be blocked may be quite common.

M.D. Y. thanks Professor Alex Keynan for his kind hospitality, and Dr Yoram Milner for generously providing laboratory space. This work was done during M.D.Y.'s tenure of a short-term fellowship of the European Molecular Biology Organization, and was supported by a grant from the Thyssen Foundation to Professor Keynan.

\section{REFERENCES}

Dancer, B. N. \& Mandelstam, J. (1981). Complementation of sporulation mutations in fused protoplasts of Bacillus subtilis. Journal of General Microbiology 123, 17-26.

Jenkinson, H. F., SAwyer, W. D. \& Mandelstam, J. (1981). Synthesis and order of assembly of spore coat proteins in Bacillus subtilis. Journal of General Microbiology 123, 1-16.

Piggor, P. J. \& CoOTE, J. G. (1976). Genetic aspects of bacterial endospore formation. Bacteriological Reviews 40, 908-962.
YudKIN, M. D., Boschwitz, H., LoRCH, Y. \& KeYNAN, A. $(1982 a)$. Changes in the pattern of protein synthesis during the first three hours of sporulation in Bacillus subtilis. Journal of General Microbiology 128, 2165-2177.

Yudkin, M. D., Boschwitz, H. \& Keynan, A. $(1982 b)$. The effect of mutations in spoOA or spoIIA on the pattern of protein synthesis in Bacillus subtilis under sporulation conditions. Molecular and General Genetics 187, 244-247. 